\title{
Facial EMG responses to dynamic emotional facial expressions in boys with disruptive behavior disorders
}

\author{
Minet de Wied ${ }^{\mathrm{a}, *}$, Anton van Boxtel ${ }^{\mathrm{b}}$, Ruud Zaalberg ${ }^{\mathrm{c}}$, \\ Paul P. Goudena ${ }^{\text {a }}$, Walter Matthys ${ }^{\mathrm{d}}$ \\ a Department of Child and Adolescent Studies, Utrecht University, Utrecht, The Netherlands \\ ${ }^{\mathrm{b}}$ Department of Psychology, Tilburg University, Tilburg, The Netherlands \\ ${ }^{c}$ Department of Psychology, University of Amsterdam, Amsterdam, The Netherlands \\ d Department of Child and Adolescent Psychiatry, and Rudolf Magnus Institute of Neuroscience, \\ University Medical Center Utrecht, Utrecht, The Netherlands
}

Received 21 April 2005; received in revised form 1 July 2005; accepted 3 August 2005

\begin{abstract}
Based on the assumption that facial mimicry is a key factor in emotional empathy, and clinical observations that children with disruptive behavior disorders (DBD) are weak empathizers, the present study explored whether DBD boys are less facially responsive to facial expressions of emotions than normal controls. Facial electromyographic (EMG) activity in the zygomaticus major and corrugator supercilii muscle regions, and heart rate activity were studied in 22 clinically referred 8-12-year-old DBD boys and 22 age-matched normal controls during exposure to dynamic happy and angry expressions. Dispositional emotional empathy was assessed by a self-report questionnaire for children. The happy and angry facial expressions evoked distinct facial EMG response patterns, with increased zygomaticus muscle activity to happy expressions and increased corrugator muscle activity to angry expressions. The corrugator (but not the zygomaticus) muscle response pattern was less pronounced for DBD boys than the normal controls. Attending to the emotional expressions was associated with equivalent cardiac deceleration in both groups, reflecting a similar orienting/attention response. Lower empathy scores were obtained for DBD boys than for normal controls. In conclusion, facial mimicry responses to angry facial expressions were subnormal in DBD boys, which may be a sign of a deficient early component in the process of emotional empathy, and thus play a role in impaired empathic responding.
\end{abstract}

(C) 2005 Elsevier Ltd. All rights reserved.

Keywords: Facial EMG; Conduct disorder; Oppositional defiant disorder; Mimicry; Empathy

\section{Introduction}

In clinical practice, children with oppositional defiant disorder (ODD) and conduct disorder (CD), also called disruptive behavior disorders (DBD), are thought to be poor in empathic skills, that is, the ability to understand and share another's emotional state (Cohen and Strayer, 1996). The clinical impression that DBD children are weak empathizers is given support by studies reporting

\footnotetext{
* Corresponding author. Tel.: +31 30 2537693; fax: +31 302537731.

E-mail address: m.dewied@fss.uu.nl (M.de Wied).
}

empathy deficits in clinically identified CD adolescents (Cohen and Strayer, 1996), and DBD boys (de Wied et al., 2005). Starting from the assumption that facial mimicry is a fundamental component in the process of empathy, especially emotional empathy (i.e., shared feelings) (Hatfield et al., 1994; Hoffman, 2000; Meltzoff, 1993), the present study explored facial electromyographic (EMG) responses in clinically referred 8-12year-old DBD boys and normal controls, when exposed to dynamic emotional facial expressions.

An early report by Lipps (1905) first called attention to the possible role of motor mimicry/imitation in the 
automatic transmission of emotions. Lipps proposed that people tend to mimic the facial, vocal or postural expressions of emotions displayed by an interaction partner, and that such mimicry responses may evoke corresponding emotions in the observer. Many researchers currently consider motor mimicry as the very essence of emotional empathy, likely to be biologically "hardwired" (e.g., Hatfield et al., 1994; Hoffman, 2000; Meltzoff, 1993; Preston and de Waal, 2002).

According to Hoffman's (2000) developmental model of empathy, mimicry is an early component in the process of empathy. The tendency to automatically mirror emotional expressions becomes manifest already in the first days of life by reflexive crying in response to other babies' crying. Primitive mechanisms (e.g., mimicry) contribute to the development of empathy in the early preverbal period, but continue to operate past childhood. As the cognitive system develops, higherorder cognitive processes (e.g., role-taking) come to play a more important role. However, mature empathic responses are generated both by primitive (automatic) mechanisms and more sophisticated cognitive processes.

Focusing on facial imitation, Dimberg and colleagues (Dimberg, 1982, 1988, 1990; Dimberg and Lundquist, 1990) demonstrated that exposure to pictures of happy and angry faces evoke distinct facial EMG response patterns. Adult subjects react spontaneously with increased zygomaticus major (cheek) muscle activity when exposed to happy facial expressions, whereas angry facial expressions evoke increased corrugator supercilii (eyebrow) muscle activity. These facial EMG reactions are rapidly evoked (i.e., under $400 \mathrm{~ms}$; Dimberg and Thunberg, 1998), also when subjects are unconsciously exposed to facial stimuli (Dimberg et al., 2000), and hard to restrain voluntarily (Dimberg et al., 2002). The data support the hypothesis that facial mimicry is an early, automatic response to others' facial displays.

Sonnby-Borgström and colleagues (Sonnby-Borgström, 2002; Sonnby-Borgström et al., 2003) conducted studies on facial EMG (zygomaticus and corrugator) and empathic sensitivity in high and low empathic students. When pictures of happy and angry expressions were presented at short exposure times (eliciting automatic reactions), the high-empathy group showed a stronger mimicry response than the low-empathy group. Furthermore, a significant correspondence between facial reactions and subjective feelings was found only among the high empathic students (Sonnby-Borgström, 2002), suggesting that those who show a stronger tendency to mimic other's facial expressions are more susceptible to emotional empathy.

The aim of the present study was to determine facial responsiveness in DBD boys and normal controls. Respondents were exposed to 5-s moving pictures showing a male model producing dynamic angry or happy facial expressions. We used dynamic presentations because moving images of facial expressions may constitute a stronger eliciting stimulus than static displays (Wehrle et al., 2000). Facial EMG activity in the zygomaticus major and corrugator supercilii muscle regions was assessed during exposure to the facial stimuli. Dispositional emotional empathy was assessed by a selfreport questionnaire for children. Based on theory and empirical studies with adults, it was predicted that happy faces would evoke more zygomaticus activity than angry faces, whereas angry faces would evoke more corrugator activity than happy faces. In fact, corrugator activity tends to be inhibited during exposure to happy faces (Dimberg and Lundquist, 1990; Dimberg and Thunberg, 1998; Dimberg et al., 2000). Furthermore, it was predicted that this typical response pattern (i.e., increased zygomaticus activity during happy faces and increased corrugator activity during angry faces) would be less pronounced for DBD boys than for normal controls. Given the preliminary nature of this first study on facial EMG responses in DBD boys, no more specific hypotheses were formulated.

Attending to affective pictures or film clips is generally associated with cardiac deceleration (McManis et al., 2001; Waldstein et al., 2000), which may be reflective of an orienting or attention response (Cook and Turpin, 1997). Cardiac deceleration was also demonstrated during exposure to happy and angry facial expressions (Dimberg, 1982, 1990). To control for differences in heart rate responses during happy versus angry facial expressions, heart rate was also measured in the present study.

\section{Methods}

The Medical Ethics Committee of the University Medical Center Utrecht approved the study protocol, and parents gave written informed consent prior to participation.

\subsection{Participants}

In the present study, data were collected within the context of a larger study on empathy in DBD boys. Only boys were included because DBD is more prevalent among boys than girls (Maughan et al., 2004). The DBD group consisted of 8-12-year-old boys who had met the criteria for ODD $(n=21)$ or CD $(n=4)$ as set out in DSM-IV (American Psychiatric Association, 1994). In total, 25 DBD boys were recruited from inpatient $(n=12)$ and day-treatment $(n=12)$ units of the Department of Child and Adolescent Psychiatry, University Medical Center Utrecht, and from a special school for children with severe behavioral disorders $(n=1)$. Because the data of three participants were lost 
due to equipment malfunction $(n=1)$ and artifacts $(n=2)$, the final DBD group contained 22 boys.

Clinical diagnoses of all DBD boys were based on extensive psychiatric and psychological assessment of the child, interviews with the parents including discussion of the developmental history, and information from the child's teacher. The clinical diagnosis was checked by the Diagnostic Interview Schedule for Children (DISCIV) (Shaffer et al., 2000). Except for the parents of three children who could not be interviewed at the time, all parents were interviewed by trained graduate students to collect DISC data. The DISC confirmed the clinical diagnoses in 18 out of 19 checked cases. The DISC also confirmed the comorbid clinical diagnosis of attention deficit-hyperactivity disorder (ADHD; $n=8$ ), in 6 out of 7 checked cases. Thirteen DBD boys were on psychopharmacological treatment, specifically on methylphenidate $(n=8)$ or risperidone $(n=5)$.

In total, 30 age-matched normal control boys were recruited from regular elementary schools in the vicinity of Utrecht. To control for behavioral problems, their parents were subjected to the DISC. In addition, the Child Behavior Checklist (CBCL) (Achenbach, 1991a) and the Teacher's Report Form (TRF) (Achenbach, 1991b), in the Dutch translation (Verhulst et al., 1996, 1997), were completed by the children's parents and teachers, respectively. Exclusion criteria for the normal controls were: (1) a DISC diagnosis of ODD or CD, and/or (2) scores on both the CBCL and TRF in the clinical range for Total Behavior Problems $(T>63)$. Conforming to these criteria, six participants were removed from the sample. In addition, the data of two participants were lost, due to equipment malfunction $(n=1)$ and artifacts $(n=1)$. As a result, the final control group contained 22 boys. None of the normal controls were on psychopharmacological treatment.

The parents and teachers of the DBD boys also completed the CBCL and TRF. Aggressive, Delinquent, and Externalizing behaviors on the CBCL and TRF were employed as measures of disruptive behavior and used to confirm group differences. Furthermore, to establish the IQ of the participating boys, the normal controls completed two subtests of the Wechsler Intelligence Scale for Children-Revised (WISC-R) (Wechsler, 1974), namely Vocabulary and Block Design. These subtests have a correlation of 0.90 with the full-scale IQ (Sattler, 1992). Full-scale IQ data of all DBD boys were collected as part of the screening procedure at intake. All participants included in the study received IQ scores $>80$.

Descriptive characteristics of the participant samples are included in Table 1. In the interest of completeness of reporting on the sample, the table also includes CBCL and TRF internalizing behavior, anxiety/depression, and attention problem scores. To examine differences between groups, the data were subjected to independent-samples $t$-tests (two-tailed). The statistical analyses revealed no age difference, nor a difference in intelligence between DBD boys and normal controls. The $t$-tests performed on the CBCL and TRF aggressive, delinquent, and externalizing scores demonstrated expected differences between groups, such that DBD boys obtained higher scores on all measures of disruptive behavior. However, DBD boys also differed from normal controls on internalizing behavior, anxiety/ depression, and attention problems.

Table 1

Characteristics of boys with disruptive behavior disorders (DBD) and normal controls

\begin{tabular}{|c|c|c|c|c|c|}
\hline & \multicolumn{2}{|c|}{ Normal controls $(n=22)$} & \multicolumn{2}{|c|}{$\operatorname{DBD}(n=22)$} & \multirow[t]{2}{*}{$t$-values $(\mathrm{df}=42)$} \\
\hline & $M$ & SD & $M$ & SD & \\
\hline \multicolumn{6}{|l|}{ Child Behavior Checklist } \\
\hline Externalizing behavior & 46.68 & 10.40 & 76.45 & 7.79 & $-10.74^{* * *}$ \\
\hline Aggressive behavior & 52.50 & 3.50 & 79.95 & 10.62 & $-11.52^{* * *}$ \\
\hline Delinquent behavior & 53.32 & 4.81 & 72.18 & 7.22 & $-10.20^{* * *}$ \\
\hline Attention problems & 53.36 & 4.60 & 68.95 & 9.74 & $-6.79^{* * *}$ \\
\hline Internalizing behavior & 54.50 & 8.53 & 70.59 & 7.65 & $-6.56^{* * *}$ \\
\hline Anxiety/depression & 56.14 & 6.40 & 71.77 & 9.02 & $-6.63^{* * *}$ \\
\hline \multicolumn{6}{|l|}{ Teacher's Report Form ${ }^{\mathrm{a}}$} \\
\hline Externalizing behavior & 46.10 & 10.14 & 68.77 & 6.54 & $-8.76^{* * *}$ \\
\hline Aggressive behavior & 52.76 & 4.54 & 69.23 & 7.37 & $-8.77^{* * *}$ \\
\hline Delinquent behavior & 53.43 & 5.55 & 65.68 & 7.72 & $-5.95^{* * *}$ \\
\hline Attention problems & 51.86 & 3.99 & 60.91 & 7.07 & $-5.14^{* * *}$ \\
\hline Internalizing behavior & 45.33 & 8.85 & 64.82 & 7.01 & $-8.03^{* * *}$ \\
\hline Anxiety/depression & 52.14 & 4.77 & 65.14 & 8.97 & $-5.89^{* * *}$ \\
\hline Age & 10.18 & 1.22 & 10.09 & 1.27 & 0.24 \\
\hline IQ & 102.86 & 13.30 & 99.14 & 12.78 & 0.95 \\
\hline
\end{tabular}

${ }^{\mathrm{a}} \mathrm{df}=41$ (scores of one normal control boy were missing).

${ }^{* * *} p<0.0001$. 


\subsection{Stimulus materials}

Emotional film clips. Two emotionally loaded film clips, each with a total duration of $5600 \mathrm{~ms}$, were made in our institute for use in the present study. A 38-yearold trained male actor portrayed expressions of happiness and anger. Both expressions were based on the Facial Action Coding System (FACS) (Ekman and Friesen, 1978). The FACS provides a detailed description of the muscular basis and the outward manifestation of human (emotional) facial behavior in terms of so-called action units (AUs). The portrayal of happiness consisted of AU6 (cheek raiser) and AU12 (lip corner puller). The portrayal of anger consisted of AU4 (brow lowerer), AU7 (lid tightener), AU17 (chin raiser), AU23 (lip tightener), and AU24 (lip pressor). A certified FACS coder coded the stimulus material on two occasions separated by a two-year period. Intra-coder reliability was calculated using the following index of agreement: Number of AUs coded on both occasions $\times 2$ and divided by the total number of Aus (see FACS). The intra-coder reliability was $100 \%$. Each film clip started with a $2000 \mathrm{~ms}$ still of the actor's neutral face (i.e., no facial activity), followed by a $2600 \mathrm{~ms}$ dynamic happy or angry expression, and ended with a $1000 \mathrm{~ms}$ neutral still. The $2000 \mathrm{~ms}$ still preceding the dynamic expression served as baseline to exclude possible confounding of reactions to the stimulus person with reactions to emotional displays.

Neutral film clip. A third neutral film clip with a total duration of $4000 \mathrm{~ms}$, showed the same male model with a neutral facial expression. The neutral face stimulus served two purposes: first, to examine the temporal profile of facial EMG activity and HR as a function of attending to the film clips per se, and second, to familiarize the participants with the stimulus person shown in the emotionally loaded film clips.

\subsection{Apparatus and physiological recordings}

Participants were individually tested at the clinic in a dimly lit room equipped with two personal computers, one for stimulus presentation and one for online control of data recording. Both computers were connected to a small portable digital data recorder (Vitaport II system) that was attached to the participant's chair. All film clips were presented using a personal computer with a 17-in. color monitor (ILYAMA A705MT). A program written with Authorware 5 Attain was used to control stimulus presentation and to generate signals marking on- and offset of each stimulus. The marker signals were stored on the Vitaport II system.

Bipolar EMG recordings (bandwidth $20-512 \mathrm{~Hz}$ ) were made from the left zygomaticus and corrugator regions of the face (Fridlund and Cacioppo, 1986) using surface $\mathrm{Ag} / \mathrm{AgCl}$ electrodes ( $10 \mathrm{~mm}$ diameter) filled with
Ten 20 conductive EEG paste. Before the electrodes were attached, the skin was cleaned and slightly rubbed with alcohol. The electrocardiogram (ECG) was recorded with disposable electrodes on the chest (sternum-V6 lead; bandwidth 5-30 Hz). The EMG and ECG signals were relayed through shielded cables to the Vitaport II system where they were digitized and stored (sample rate: $1024 \mathrm{~Hz}$ ). ECG R-waves were detected offline and intervals between heart beats were converted into heart rate (HR), expressed in beats per minute. A procedure was applied for correction of long and short heart beat intervals that were caused by erroneous $\mathrm{R}$-wave detection due to excessive movement (Stekelenburg and van Boxtel, 2001). EMG was bandreject filtered (bandwidth $48-52 \mathrm{~Hz}$ ) to remove any influence of 50 $\mathrm{Hz}$ power line interference. Data were then visually inspected for remaining artifacts, resulting in the exclusion of three participants from subsequent analysis, as indicated above.

\subsection{Dispositional emotional empathy}

Bryant's (1982) Index of Empathy for Children and Adolescents (IECA) was used to assess empathic tendencies. The 22-item questionnaire has been designed to assess emotional responsiveness, rather than cognitive insight. The items tap a range of affective reactions (e.g., "Seeing a (girl/boy) crying makes me feel like crying", "It makes me sad to see a (girl/boy) who can't find anyone to play with"). Bryant (1982) has demonstrated satisfactory test-retest reliability and construct validity of the IECA. The present study used the IECA in a Dutch translation, with the child two-point (yes-no) response format. Children were asked to agree or disagree with each of the 22 statements. Empathic answers were assigned the value 1 , non-empathic answers the value 0 . An emotional empathy score was computed by adding the 22 item scores $(0-22)$. The scores were subjected to an independent-samples $t$-test (one tailed).

\subsection{Procedure}

Participants were received in the test room by one of four female experimenters. The experimenters were trained and used written protocols detailing the regular procedure and verbal instructions. Prior to the test session, the control boys completed the two WISC-R subtests in a separate room. Upon completion, the child was guided back to the test room where he was seated in a chair. After the electrodes were attached to the participant's face and chest, the empathy questionnaire was administered. The experimenter read the 22 items aloud, and entered the child's answers on a sheet. The participant was then seated at a table facing the monitor of the stimulus computer at approximately $80-\mathrm{cm}$ distance. 
To foster relaxation and adaptation to the testing environment, participants were first exposed to a 5min aquatic film clip (Piferi et al., 2000). After the lights were dimmed, the participant was instructed to relax and enjoy the clip. Next, the participant was told that he would see a male model producing facial expressions of emotion. To conceal the main purpose, the experimenter explained that we just wanted to check whether the expressions looked familiar, and that he would be asked to name the expressions. The happy and angry film clips were repeated four times in succession (interstimulus interval: $6 \mathrm{~s}$ ), and shown in counterbalanced order. The neutral film clip was presented prior to each sequence of emotional film clips (interstimulus interval: $4 \mathrm{~s}$ ). The participant was asked to name the expression after each sequence of happy and angry expressions (nearly all participants correctly identified the emotional expressions). During film presentation the experimenter remained in the same room outside the child's scope of vision, seated in front of the observation monitor.

\subsection{Statistical analysis of physiological responses}

To examine the temporal profiles of facial EMG during the neutral film clip, mean EMG amplitudes during subsequent $100-\mathrm{ms}$ time intervals were expressed as a percentage of the mean amplitude during the entire 4000-ms stimulus. Percentage scores were introduced to standardize the widely differing absolute EMG amplitudes of individual participants and, thus, to enable comparison between individuals and groups. Mean HR was calculated during each 500 -ms time interval of the same stimulus.

Repeated measures MANOVAs were performed on the EMG percentage scores with intervals (40 100-ms intervals) and clip presentation (twofold) as within-subjects factors, and group (DBD vs. controls) as a between-subjects factor. To examine the temporal profile of HR during the same stimulus, a repeated measures MANOVA was conducted, with intervals (8 500-ms intervals) and clip presentation (twofold) as within-subjects factors, and group (DBD vs. controls) as a between-subjects factor.

To examine facial EMG responses to happy versus angry faces, mean EMG amplitudes during the 2600ms happy and angry expressions were expressed as a percentage of the mean amplitude during the preceding 2000-ms still of the actor's neutral face (baseline period). HR responses were expressed as difference scores, that is, the difference in mean HR between the 2600 -ms emotional expressions and the 2000-ms baseline period.

Repeated measures MANOVAs were performed on the EMG percentage scores with emotional expression (happy vs. angry) and trials (4) as within-subjects factors, and group (DBD vs. controls) as a betweensubjects factor. A similar MANOVA was conducted on HR difference scores. One-sample $t$-tests (one-tailed) were further employed to examine changes from baseline in facial EMG activity and HR for each group, and for each emotional expression. A significance level of 0.05 was used in all analyses.

\section{Results}

Prior to conducting the main analyses of the study, the physiological (facial EMG and HR) and self-report (emotional empathy) data were subjected to independent-samples $t$-tests (two-tailed) comparing DBD boys who were on medical treatment with DBD boys who were not; DBD boys with and without ADHD; and DBD boys from inpatient units with those from daytreatment units. Except for a significant difference in HR response during angry faces between DBD boys with and without $\operatorname{ADHD}\left(t_{20}=-2.53, p<0.05\right)$, no significant differences emerged on any of the physiological response measures, nor on emotional empathy. Therefore, the scores of all DBD subjects were collapsed in subsequent analyses.

\subsection{Temporal profiles of facial EMG and HR during the neutral face film clip}

Facial EMG. For both muscle regions, the MANOVAs revealed no significant effect of intervals, nor significant intervals $\mathrm{X}$ group, and intervals $\mathrm{X}$ presentation interactions, indicating a flat profile in zygomaticus and corrugator EMG activity throughout the $4000 \mathrm{~ms}$ neutral face stimulus for each stimulus presentation within both groups. Hence, differences in facial EMG activity during the emotional expressions cannot be attributed to the effects of attending to the film clips as such. The temporal profiles for both muscle regions during the neutral film clip (collapsed across presentations) are illustrated in Fig. 1.

$H R$. The MANOVA revealed a significant effect of intervals $\left(F_{7,36}=9.84, p<0.0001\right)$, but no significant intervals $\mathrm{X}$ group, or intervals $\mathrm{X}$ presentation interaction. HR significantly decreased across the eight time intervals, probably reflecting an orienting or attention response. Moreover, the analysis revealed a significant effect of group $\left(F_{1,42}=15.85, p<0.001\right)$, with higher HR values for $\mathrm{DBD}$ boys $($ mean $=88.29, \mathrm{SD}=10.23)$ than for normal controls (mean $=76.23, \mathrm{SD}=9.86$ ). It is important to note that higher HR values were also observed in the DBD group (mean $=89.79, \mathrm{SD}=9.66)$ relative to the control group $($ mean $=75.16, \mathrm{SD}=9.01)$ during the aquatic film clip $\left(t_{42}=-5.19, p<0.0001\right)$. Since DBD boys were rated by parents and teachers as more anxious than controls (Table 1), it is possible that they exhibited higher HR values because they experienced more anxiety in a novel laboratory situation. 

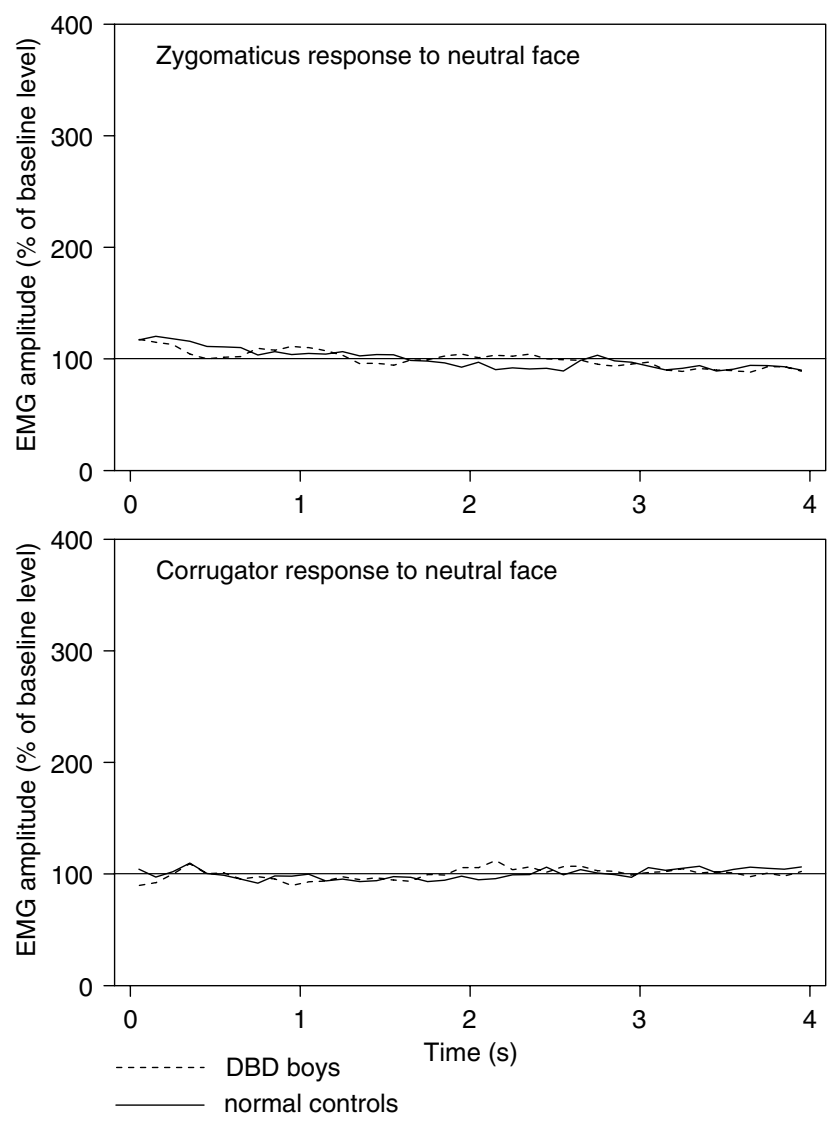

Fig. 1. Mean EMG activity of zygomaticus and corrugator for DBD boys and normal controls during presentation of the neutral face film clip.

\subsection{Emotional empathy}

Consistent with predictions, an independent-samples $t$-test yielded a significant difference between groups $\left(t_{42}=2.04, p<0.05\right)$, with lower empathy scores for DBD boys $($ mean $=10.77, \mathrm{SD}=2.89)$ than controls (mean $=12.55, \mathrm{SD}=2.87)$.

\subsection{Facial EMG responses to dynamic emotional facial expressions}

Zygomaticus. The MANOVA ${ }^{1}$ revealed a significant effect of emotional expression $\left(F_{1,42}=13.26\right.$, $p<0.001$ ), but no significant effect of group or trials, nor significant interactions. Consistent with predictions, happy faces evoked a significantly larger increase in zygomaticus EMG activity than angry faces (Fig. 2).

Since trials had no significant effect, the data were collapsed across the four trials, and subjected to onesample $t$-tests to further evaluate changes from baseline within each group. The analyses revealed a significant

\footnotetext{
${ }^{1}$ A MANCOVA with clinically diagnosed ADHD as a dichotomous covariate yielded similar results.
}
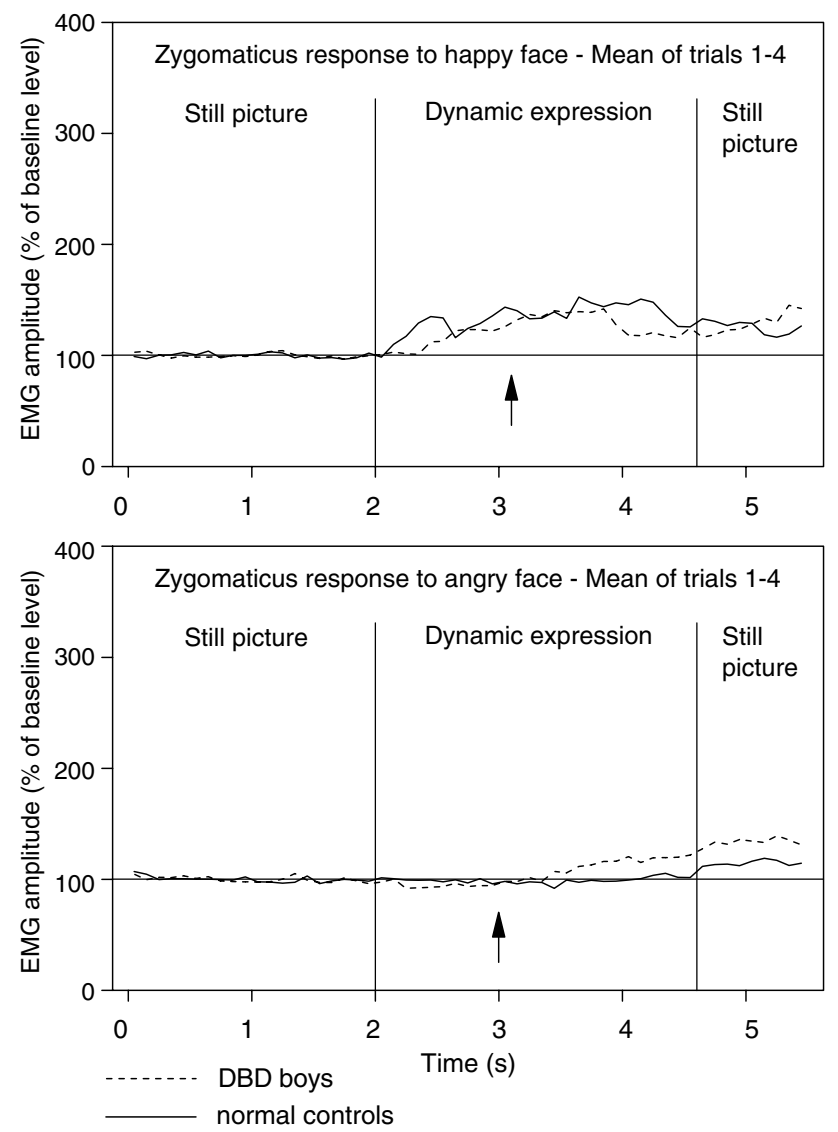

Fig. 2. Mean zygomaticus EMG responses for DBD boys and normal controls to dynamic emotional facial expressions collapsed across presentation trials. Arrows indicate the moment at which maximal expression is attained.

increase from baseline level in zygomaticus EMG activity during exposure to happy expressions, both for DBD boys $\left(t_{21}=3.30, \quad p<0.01\right)$, and normal controls $\left(t_{21}=3.45, p<0.01\right)$. There was no significant change from baseline level during exposure to angry expressions.

Corrugator. The MANOVA ${ }^{1}$ yielded significant effects of emotional expression $\left(F_{1,42}=35.49\right.$, $p<0.0001)$ and group $\left(F_{1,42}=4.50, p<0.05\right)$, but not of trials. Consistent with predictions, angry faces evoked a significantly larger increase in corrugator EMG activity than happy faces, which, in turn, were associated with EMG inhibition (Fig. 3). Also consistent with predictions, the EMG response during angry faces was smaller for DBD boys than for normal controls. Posthoc $t$-tests (one-tailed) revealed a significant group difference for angry faces $\left(t_{42}=1.89, p<0.05\right)$, but not for happy faces. Except for a significant emotional expression $\mathrm{X}$ trials interaction $\left(F_{3,40}=3.30, p<0.05\right)$, the analysis yielded no significant interaction effects. This interaction can be attributed to the fact that corrugator EMG activity showed a linearly diminishing inhibition across the four presentations of happy expressions $\left(F_{1,43}=11.82, p<0.01\right)$, but tended to show 

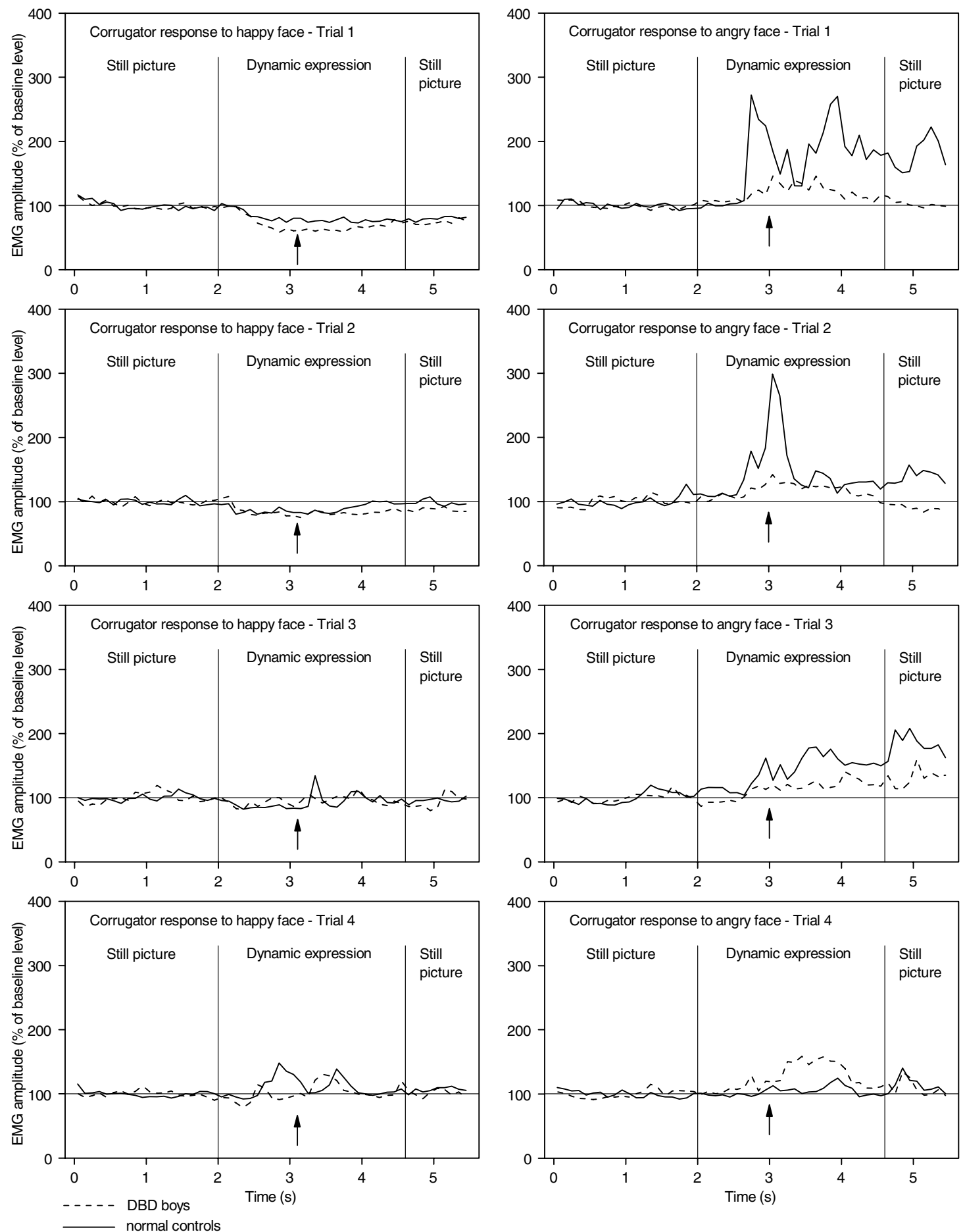

Fig. 3. Mean corrugator EMG responses for DBD boys and normal controls to dynamic emotional facial expressions for separate presentation trials. Arrows indicate the moment at which maximal expression is attained.

a linear reduction in facilitation across the four presentations of angry expressions $\left(F_{1,43}=2.88, p<0.1\right)$. These findings suggest that subjects became habituated to the repeatedly presented stimuli.

Accordingly, one-sample $t$-tests were conducted for each group at each trial, to examine changes from baseline level. The normal control group showed a signifi- cant increase in corrugator EMG activity from baseline during exposure to angry expressions at the first $\left(t_{21}=2.38, p<0.05\right)$, second $\left(t_{21}=2.28, p<0.05\right)$ and third trial $\left(t_{21}=2.79, p<0.01\right)$, but not at the fourth trial. The pattern was slightly different for the DBD group. DBD boys showed a significant increase at the first $\left(t_{21}=2.44, p<0.05\right)$, second $\left(t_{21}=2.55, p<0.01\right)$, 
and fourth trial $\left(t_{21}=2.10, p<0.05\right)$, but not at the third trial. Furthermore, corrugator showed a significant decrease in EMG activity from baseline during exposure to happy expressions for both groups on the first (controls, $t_{21}=-4.86, \quad p<0.0001 ; \quad \mathrm{DBD}, t_{21}=-6.16$, $p<0.0001$ ) and second (controls, $t_{21}=-2.23$, $p<0.05$; DBD, $\left.t_{21}=-4.00, p<0.001\right)$ trial, but not on the last two trials. The overall pattern shows that activity in corrugator is potentiated by angry expressions and inhibited by happy expressions. These findings match well with earlier findings (Larsen et al., 2003), demonstrating a linear effect of bipolar emotional valence on corrugator (but not on zygomaticus) EMG activity, with corrugator activity being potentiated by unpleasant pictures and inhibited by pleasant pictures.

\subsection{HR responses to dynamic emotional facial expressions}

The MANOVA ${ }^{1}$ yielded no significant main effects, nor significant interactions, indicating that the happy and angry faces evoked quite similar HR responses across and within groups. One-sample $t$-tests further demonstrated significant cardiac decelerations during angry and happy faces in both groups, specifically, in the normal control group during angry $\left(t_{21}=-4.36\right.$, $p<0.001)$ and happy $\left(t_{21}=-3.60, p<0.001\right)$ expressions, as well as in the DBD group during angry $\left(t_{21}=-4.92, \quad p<0.0001\right)$ and happy $\left(t_{21}=-3.60\right.$, $p<0.001)$ expressions. These findings suggest that both expressions equally attracted attention.

\section{Discussion}

Before addressing the principal findings concerning facial EMG in DBD boys and normal controls, it is important to note that DBD boys scored significantly lower on a self-report measure of emotional empathy than normal controls. These findings support clinical observations that DBD children are weak empathizers.

The purpose of the current study was to examine facial responsiveness in DBD boys and normal controls. We demonstrated that angry and happy facial stimuli spontaneously elicit different EMG response patterns. Angry faces evoked a stronger increase in corrugator activity than happy faces, while happy faces evoked a stronger increase in zygomaticus activity than angry faces. These findings replicate data obtained in studies with adults in normal populations (Dimberg, 1982, 1988, 1990; Dimberg and Lundquist, 1990). Furthermore, as predicted, the corrugator response to angry faces was less pronounced for DBD boys than for normal controls. However, no significant difference between groups emerged in the zygomaticus response to happy faces. The present findings match well with studies indi- cating that facial expressivity to negative slides (but not to positive slides) relates negatively to externalizing behaviors for boys (Eisenberg et al., 2001; Zhou et al., 2002). In the present study, DBD boys showed a smaller corrugator EMG response, a muscle region principally associated with negative emotions.

These findings demonstrate that DBD boys are less facially responsive than normal controls towards angry faces. Assuming that facial imitation is a factor in the process of emotional empathy, it can be proposed that deficits in facial reactivity may play a role in DBD boys' impaired empathic responding. However, it is uncertain whether the present findings extend to other negative emotions most relevant to empathy and prosocial responding, namely fear and sadness, or whether they are exclusively associated with facial expressions of anger. Research suggests that children with antisocial behavior problems show biases and deficits in anger encoding and interpretation. For example, they focus more on aggressive cues in the environment (Gouze, 1987), tend to misinterpret emotions as anger (Cadesky et al., 2000), and are inclined to make hostile attributions (Quiggle et al., 1992). Accordingly, further research is needed to examine facial EMG responses in DBD children to a larger range of emotional facial expressions.

Facial mimicry is thought to have a biologically 'prewired' neural basis (e.g., Dimberg et al., 2002; Preston and de Waal, 2002). Since relatively long exposure times were used in the present study, it is not possible to decide whether the present facial EMG patterns reflect preconscious mimicking behavior or facial expressions as a readout of inner feeling states. However, in agreement with the 'innate basis' hypothesis, distinct facial reactions emerged already in the first second of exposure to dynamic facial expressions. Within corrugator, early responses to angry expressions decreased over repeated trials, reflecting habituation which is typical of reflexive responses. There may be a relationship with the fast habituation of amygdalar responses to emotional facial expressions (Breiter et al., 1996; Whalen et al., 1998), in particular because the amygdala are involved in relatively fast, automatic facial mimicry reactions (Wild et al., 2003). Interestingly, as seen in Fig. 3, habituation to angry faces is clearly visible for normal controls, but not for DBD boys. No significant interaction between emotional expression, group, and trials was observed, however, so prudence is called for interpreting these graphs. Nevertheless, the pattern calls attention to possible impairments in one of the simplest forms of information processing among DBD children, which certainly deserves further investigation.

To our knowledge, this is the first study using facial EMG to examine facial responsiveness in school-aged children with disruptive behavior problems and normal controls. These two groups showed differences in corru- 
gator EMG responses, with DBD boys being less facially responsive than normal controls towards angry faces. Assuming that facial imitation is a factor in the process of emotional empathy, it can be proposed that deficits in facial reactivity may play a role in DBD boys' impaired empathic responding. Given the limitations of the current study, however, this interpretation should be viewed as provisional awaiting further research.

\section{Acknowledgements}

We thank Jocelyne Posthumus and Mariette Rietsema for assistance with data collection, Ton Aalbers for the development of data analysis software, and Peter Bunck for technical assistance.

\section{References}

Achenbach TM. Manual for the child behavior checklist and 1991 profile. Burlington, VT: University of Vermont, Department of Psychiatry, 1991.

Achenbach TM. Manual for the teacher's report form and 1991 profile. Burlington, VT: University of Vermont, Department of Psychiatry; 1991.

American Psychiatric Association. Diagnostic and statistical manual of mental disorders, 4th ed. (DSM-IV). Washington, DC: American Psychiatric Press; 1994.

Breiter HC, Etcoff NL, Whalen PJ, Kennedy WA, Rauch SL, Buckner $\mathrm{RL}$, et al.. Response and habituation of the human amygdala during visual processing of facial expression. Neuron 1996;17:875-87.

Bryant BK. An index of empathy for children and adolescents. Child Development 1982;53:413-25.

Cadesky EB, Mota VL, Schachar RJ. Beyond words: How do children with ADHD and/or conduct problems process nonverbal information about affect? Journal of the American Academy of Child and Adolescent Psychiatry 2000;39:1160-7.

Cohen D, Strayer J. Empathy in conduct-disordered and comparison youth. Developmental Psychology 1996;32:988-98.

Cook EW, Turpin G. Differentiating orienting, startle, and defense responses: the role of affect and its implications for psychopathology. In: Lang PJ, Simons RF, Balaban MT, editors. Attention and orienting: sensory and motivational processes. Mahwah, NJ: Erlbaum; 1997. p. 137-64.

De Wied M, Goudena PP, Matthys W. Empathy in boys with disruptive behavior disorders. Journal of Child Psychology and Psychiatry 2005;46:867-80.

Dimberg U. Facial reactions to facial expressions. Psychophysiology 1982;19:643-7.

Dimberg U. Facial electromyography and the experience of emotion. Journal of Psychophysiology 1988;2:277-82.

Dimberg U. Facial electromyography and emotional reactions. Psychophysiology 1990;27:481-94.

Dimberg U, Lundquist LO. Gender differences in facial reactions to facial expressions. Biological Psychology 1990;30:151-9.

Dimberg U, Thunberg M. Rapid facial reactions to emotional facial expressions. Scandinavian Journal of Psychology 1998;39:39-45.

Dimberg U, Thunberg M, Elmehed K. Unconscious facial reactions to emotional facial expressions. Psychological Science 2000;11:86-9.

Dimberg U, Thunberg M, Grunedal S. Facial reactions to emotional stimuli: automatically controlled emotional responses. Cognition and Emotion 2002;16:449-71.
Eisenberg N, Losoya S, Fabes RA, Guthrie IK, Reiser M, Murphy B, et al.. Parental socialization of children's dysregulated expression of emotion and externalizing problems. Journal of Family Psychology 2001;15:183-205.

Ekman P, Friesen WV. The Facial Action Coding System. Palo Alto, CA: Consulting Psychologists Press; 1978.

Fridlund AJ, Cacioppo JT. Guidelines for human electromyographic research. Psychophysiology 1986;23:567-89.

Gouze KR. Attention and social problem solving as correlates of aggression in preschool males. Journal of Abnormal Child Psychology 1987;15:181-97.

Hatfield E, Cacioppo JT, Rapson RL. Emotional contagion. Cambridge: Cambridge University Press; 1994.

Hoffman ML. Empathy and moral development. Cambridge: Cambridge University Press; 2000.

Larsen JT, Norris CJ, Cacioppo JT. Effects of positive and negative affect on electromyographic activity over zygomaticus major and corrugator supercilii. Psychophysiology 2003;40:776-85.

Lipps T. Das Wissen von fremden Ichen. In: Lipps $T$, editor. Psychologische Untersuchungen. Leipzig: Wilhelm Engelmann; 1905. p. 697-722.

Maughan B, Rowe R, Messer J, Goodman R, Meltzer H. Conduct disorder and oppositional disorder in a national sample: developmental epidemiology. Journal of Child Psychology and Psychiatry 2004;45:609-21.

McManis MH, Bradley MM, Berg WK, Cuthbert BN, Lang PJ. Emotional reactions in children: verbal, physiological, and behavioral responses to affective pictures. Psychophysiology 2001;38:222-31.

Meltzoff AN. The centrality of motor coordination and proprioception in social and cognitive development: from shared actions to shared minds. In: Savelsbergh GJP, editor. The development of coordination in infancy. Amsterdam: Elsevier Science Publishers; 1993. p. $463-96$.

Piferi RL, Kline KA, Younger J, Lawler KA. An alternative approach for achieving cardiovascular baseline: viewing an aquatic video. International Journal of Psychophysiology 2000;37:207-17.

Preston SD, de Waal FBM. Empathy: its ultimate and proximate bases. Behavioral and Brain Sciences 2002;25:1-72.

Quiggle NL, Garber J, Panak WF, Dodge KA. Social information processing in aggressive and depressed children. Child Development 1992;63:1305-20.

Sattler JM. Assessment of children. 3rd ed. San Diego, CA: Jerome M, Sattler Publisher; 1992.

Shaffer D, Fisher P, Lucas CP, Dulcan MK, Schwab-Stone ME. NIMH Diagnostic Interview Schedule for Children Version IV (NIMH-DISC-IV): description, differences from previous versions and reliability of some common diagnoses. Journal of the American Academy of Child and Adolescent Psychiatry 2000;39:28-38.

Sonnby-Borgström M. Automatic mimicry reactions as related to differences in emotional empathy. Scandinavian Journal of Psychology 2002;43:433-43.

Sonnby-Borgström M, Jönsson P, Svensson O. Emotional empathy as related to mimicry reactions at different levels of information processing. Journal of Nonverbal Behavior 2003;27:3-23.

Stekelenburg JJ, van Boxtel A. Inhibition of pericranial muscle activity, respiration, and heart rate enhances auditory sensitivity. Psychophysiology 2001;38:629-41.

Verhulst FC, van der Ende J, Koot HM. Handleiding voor de CBCL/ 4-18 [Manual for the CBCL/4-18]. Rotterdam: Sophia Kinderziekenhuis, Erasmus University; 1996.

Verhulst FC, van der Ende J, Koot HM. Handleiding voor de Teacher's Report Form (TRF) [Manual for the Teacher's Report Form (TRF)]. Rotterdam: Sophia Kinderziekenhuis, Erasmus University; 1997. 
Waldstein SR, Kop WJ, Schmidt LA, Haufler AJ, Krantz DS, Fox NA. Frontal electrocortical and cardiovascular reactivity during happiness and anger. Biological Psychology 2000;55:3-23.

Wechsler D. Manual for the Wechsler Intelligence Scale for ChildrenRevised. New York: Psychological Corporation; 1974.

Wehrle T, Kaiser S, Schmidt S, Scherer KR. Studying the dynamics of emotional expression using synthesized facial muscle movements. Journal of Personality and Social Psychology 2000;78:105-19.

Whalen PJ, Rauch SL, Etcoff NL, McInerney SC, Lee MB, Jenike MA. Masked presentations of emotional facial expressions mod- ulate amygdala activity without explicit knowledge. Journal of Neuroscience 1998;18:411-8.

Wild B, Erb M, Eyb M, Bartels M, Grodd W. Why are smiles contagious? An fMRI study of the interaction between perception of facial affect and facial movements. Psychiatric Research: Neuroimaging 2003;123:17-36.

Zhou Q, Eisenberg N, Losoya SH, Fabes RA, Reiser M, Guthrie IK, et al.. The relations of parental warmth and positive expressiveness to children's empathy-related responding and social functioning: a longitudinal study. Child Development 2002;73:893-915. 